\title{
Resistance Trend Estimation Using Regression Analysis to Enhance Antimicrobial Surveillance: A Multi-Centre Study in London 2009-2016
}

\author{
Bernard Hernandez ${ }^{1,2, *}\left(\mathbb{D}\right.$, Pau Herrero-Viñas ${ }^{1,2}{ }^{\oplus}$, Timothy M. Rawson ${ }^{2,3} \mathbb{D}^{\circ}$, Luke S. P. Moore ${ }^{4}(\mathbb{D}$, \\ Alison H. Holmes $2,3(1)$ and Pantelis Georgiou 1,2 1
}

1 Centre for Bio-Inspired Technology, Department of Electrical and Electronic Engineering, Imperial College London, London SW7 2AZ, UK; p.herrero-vinias@imperial.ac.uk (P.H.-V.); pantelis@imperial.ac.uk (P.G.)

2 Centre for Antimicrobial Optimisation (CAMO), Imperial College London, London W12 0NN, UK; timothy.rawson07@imperial.ac.uk (T.M.R.); alison.holmes@imperial.ac.uk (A.H.H.)

3 National Institute for Health Research Health Protection Research Unit in Healthcare Associated Infections and Antimicrobial Resistance, Imperial College London, London W12 0NN, UK

4 Chelsea and Westminster NHS Foundation Trust, London SW10 9NH, UK; 1.moore@imperial.ac.uk

* Correspondence: b.hernandez-perez@imperial.ac.uk

Citation: Hernandez, B.; Herrero-Viñas, P.; Rawson, T.M.; Moore, L.S.P.; Holmes, A.H.; Georgiou, P.; Resistance Trend Estimation Using Regression

Analysis to Enhance Antimicrobial Surveillance: A Multi-Centre Study in London 2009-2016. Antibiotics 2021, 10, 1267. https://doi.org/10.3390/ antibiotics 10101267

Academic Editors: Rita Murri and Massimo Fantoni

Received: 6 September 2021 Accepted: 6 October 2021

Published: 18 October 2021

Publisher's Note: MDPI stays neutral with regard to jurisdictional claims in published maps and institutional affiliations.

Copyright: (c) 2021 by the authors. Licensee MDPI, Basel, Switzerland. This article is an open access article distributed under the terms and conditions of the Creative Commons Attribution (CC BY) license (https:/ / creativecommons.org/licenses/by/ $4.0 /)$.

\begin{abstract}
In the last years, there has been an increase of antimicrobial resistance rates around the world with the misuse and overuse of antimicrobials as one of the main leading drivers. In response to this threat, a variety of initiatives have arisen to promote the efficient use of antimicrobials. These initiatives rely on antimicrobial surveillance systems to promote appropriate prescription practices and are provided by national or global health care institutions with limited consideration of the variations within hospitals. As a consequence, physicians' adherence to these generic guidelines is still limited. To fill this gap, this work presents an automated approach to performing local antimicrobial surveillance from microbiology data. Moreover, in addition to the commonly reported resistance rates, this work estimates secular resistance trends through regression analysis to provide a single value that effectively communicates the resistance trend to a wider audience. The methods considered for trend estimation were ordinary least squares regression, weighted least squares regression with weights inversely proportional to the number of microbiology records available and autoregressive integrated moving average. Among these, weighted least squares regression was found to be the most robust against changes in the granularity of the time series and presented the best performance. To validate the results, three case studies have been thoroughly compared with the existing literature: (i) Escherichia coli in urine cultures; (ii) Escherichia coli in blood cultures; and (iii) Staphylococcus aureus in wound cultures. The benefits of providing local rather than general antimicrobial surveillance data of a higher quality is two fold. Firstly, it has the potential to stimulate engagement among physicians to strengthen their knowledge and awareness on antimicrobial resistance which might encourage prescribers to change their prescription habits more willingly. Moreover, it provides fundamental knowledge to the wide range of stakeholders to revise and potentially tailor existing guidelines to the specific needs of each hospital.
\end{abstract}

Keywords: antimicrobial resistance; resistance rate; resistance trend; antimicrobial surveillance; regression analysis; time series analysis; escherichia coli; staphylococcus aureus

\section{Introduction}

The growing threat of antimicrobial resistance (AMR) is a leading patient health and safety issue, with estimates that AMR will be responsible for more than 10 million deaths by 2050 [1]. The development of resistance in pathogens is another manifestation of the Darwinian theory of biological evolution [2] and is accelerated by circumstances where selective pressure is exerted. As a result, over the last years, research has focused 
on identifying the factors contributing to AMR [1,3,4]. The alarming outcomes have motivated the emergence of different strategies and guidelines to analyse, present and ultimately combat antimicrobial resistance. At a national level, Public Health England implemented the English surveillance program for antimicrobial utilisation and resistance (ESPAUR) which provides annual reports as a benchmark for determining appropriate local action [5]. At an international level, the European Centre for Disease Prevention and Control through the European antimicrobial resistance surveillance network (EARSNet) has created the largest publicly funded system for antimicrobial surveillance in Europe [6]. Furthermore, the World Health Organization has recently implemented the global antimicrobial resistance surveillance system (GLASS) [7] to strengthen the evidence base on AMR and inform decision-making. Unfortunately, despite of these initiatives, homogeneity of antimicrobial policies still produce different AMR outcomes [8].

The misuse and overuse of antimicrobials in humans has been identified as a major driver of AMR [3,9]. Whilst reasons for the misuse and overuse of antimicrobials are complex, a number of factors have been described. At the individual level, physicians often prioritise the management of the patient being treated, paying little regard to the long-term consequences of overusing antimicrobials [10]. Moreover, the majority of antimicrobial prescribing is performed by individuals who are not experts in infection management and may have limited understanding of antimicrobials and AMR [3,11-14]. To address the challenges posed by AMR, the importance of behaviour change interventions to improve the long-term use of antimicrobials in infection management has been recognised [15-17]. Thus, there is potential to improve the prescription behaviour of physicians through the implementation of effective communication strategies to present local rather than general AMR surveillance data.

\subsection{The Need of Local AMR Surveillance}

The guidelines on empirical antibiotic use often disregard local resistance patterns in their recommendations [18]. However, with increasing electronic recording of data, there is a growing interest in the potential secondary use of microbiological laboratory data to provide the necessary information to support antimicrobial stewardship programs (AMS) [19] which are crucial to guide health care organizations designing evidence-based policies to combat AMR [20,21]. Local susceptibility reporting and surveillance has been shown to be determinant to inform empiric antimicrobial therapy selection [22-24]. For example, a general hospital in mid-Norway reported lower antimicrobial resistance rates than the rest of countries outside Scandinavia within the blood stream infection cohort [25]. As a consequence, appropriate empiric antibiotic therapy was achieved to a larger extent by tailoring existing guidelines according to the local resistance patterns previously identified.

\subsubsection{Measuring Resistance from Susceptibility Data}

The most widespread resistance measurement is denoted as Single Antimicrobial Resistance Index (SARI) and evaluates the proportion (or percentage) of hosts harbouring resistant pathogens within a certain population. In scenarios where a pathogen exhibits resistance to numerous antimicrobials, the Multiple Antimicrobial Resistance Index (MARI) evaluates the ratio of antimicrobials to which a pathogen is resistant [26]. These metrics inform clinicians on overall antimicrobial resistance levels; however, they overlook information such as resistance tendency or seasonality. For such purpose, the previously explained indexes are computed on consecutive and independent time intervals to produce resistance time series signals. Unfortunately, these are predominantly analysed by means of visual graphs and described vaguely with expressions such as 'slightly increased', 'decreased' or 'remained constant'. The exploitation of computational algorithms to automate the handling and interpretation of large microbiology datasets is still limited [27,28]. 


\subsubsection{Evaluating Tendency on Time Series Signals}

Time series analysis comprises statistical methods to extract meaningful statistics and characteristics from time series data which is commonly split into four main components: trend, cyclical fluctuation, seasonal variation and residual effect. The trend represents tendencies and regularities in the time series and it is crucial in domains such as stock market, meteorology or biology. The methods suitable for detection and estimation of trends in a particular domain are determined by: (i) the definition of trend; (ii) the model used for the trend; (iii) the characteristics of the data; and (iv) the application objectives. Linear regression is perhaps the most popular method to estimate trends when the trend is defined as the growth rate of a population. In linear regression, the trend is expressed as a linear function of time where ordinary least squares regression has been widely used due to its simplicity to understand [29]. However, it is highly affected by outliers, non-linearity, correlation among variables and heteroskedasticity [30]. To overcome these flaws, there are alternatives such as weighted least squares regression and robust regression. On the other side, autoregressive integrated moving average (ARIMA) is widely applied in time series analysis and has proven to be robust for short term forecasting based on previous observations [31,32]. A number of variations on the ARIMA model have been developed to consider seasonal variations (SARIMA) or handle multiple time series (VARIMA).

In this study, we compare the robustness of three different regression analysis methods to quantify secular resistance trends. Moreover, the results obtained are compared with those reported in the existing literature for three different case studies: (i) Escherichia coli in urine cultures; (ii) Escherichia coli in blood cultures; and (iii) Staphylococcus aureus in wound cultures.

\section{Materials and Methods}

The methodology implemented to estimate secular trends in AMR from susceptibility data is described in Figure 1. Firstly, the microbiology data was divided into combinations, which are defined by the sample type and a pair pathogen-antimicrobial. For each combination, the resistance time series signal was generated using either independent or overlapping time intervals. The time series was linearly interpolated to fill sporadic missing values. No additional filtering was applied. An analysis of stationarity around a trend was carried out to identify those combinations fulfilling the requirements posed by ARIMA. To conclude, regression analysis was applied to quantify the tendency of the time series.

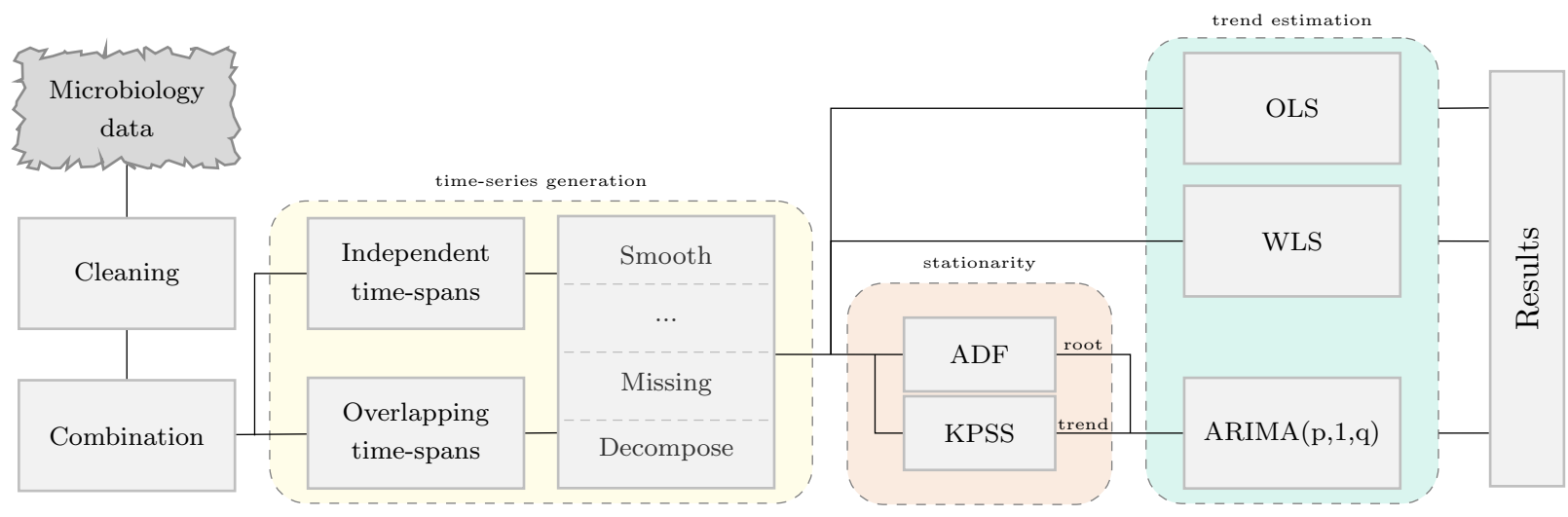

Figure 1. High-level methodology diagram. It is composed by three main sections: time series generation (yellow), stationarity analysis (orange) and trend estimation (green). The stationarity analysis was performed using the Augmented Dickey-Fuller (ADF) and the Kwiatkowski-Phillips-Schmidt-Shin (KPSS) tests to identify root-stationary and trendstationary time series signals. The regression analysis methods considered were ordinary least squares (OLS), weighted least squares (WLS) and autoregressive integrated moving average (ARIMA). 


\subsection{Microbiology Data}

This study was conducted with data from the Imperial College Healthcare NHS Trust, which comprises three separate hospitals. Data contained more than 3.5 million susceptibility tests for over 300,000 isolates corresponding to approximately 200,000 individuals. Laboratory operating procedures followed national standards for microbiological investigation [33]; isolates were identified using $\mathrm{API}^{\circledR}$ (bioMèrieux) from 2009 to 2011 and by MALDI-TOF spectroscopy (Biotyper ${ }^{\circledR}$, Brunker) from 2011 to 2015. Susceptibilities were determined by disc diffusion using BSAC criteria [34]. Duplicated or incomplete entries caused by either human (e.g., reporting same results twice accidentally) or software errors were eliminated. In addition, results were de-duplicated to discard identical organisms repeatedly isolated from a patient during the same hospital admission.

\subsection{Attributes in a Susceptibility Test Record}

Susceptibility test records are composed by laboratory number, patient number, date, sample type or culture (e.g., blood or urine), pathogen, antimicrobial, reported status and outcome (resistant, sensitive or intermediate). These were grouped for each sample type by pairs (pathogen, antimicrobial) since it is widely accepted by clinicians as detailed in the UK five year strategy in AMR [20].

\subsection{Generation of Resistance Time Series Signals}

The Single Antimicrobial Resistance Index (SARI) is stated in Equation (1) where $\mathrm{R}, \mathrm{I}$ and $\mathrm{S}$ represent the number of susceptibility tests with resistant, intermediate and susceptible outcomes respectively. It provides a value within the range $[0,1]$ where values close to one indicate high resistance.

$$
S A R I=\frac{R+I}{R+I+S} .
$$

To study the temporal evolution of AMR, it is necessary to generate a resistance time series from the susceptibility test data. This is often achieved by computing the resistance index on consecutive partitions of the data (see Table 1). The traditional strategy of dealing with partitions considers independent time intervals (see yearly, monthly or weekly time series). Unfortunately, this strategy forces to trade-off between granularity (level of detail) and accuracy. The overlapping time intervals strategy drops such dependence by defining a window of fixed size which is moved across time. The length of the window is denoted as period and the time step as shift. For instance, three time series obtained using the overlapping time intervals strategy with a monthly shift $(1 \mathrm{M})$ and window lengths of 12,6 and 3 have been presented for the sake of clarity (see 1M12, 1M6 and 1M3). The

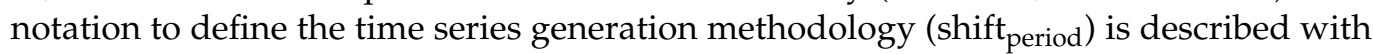
various examples in Table 1 . For instance, $7 \mathrm{D}_{4}$ defines a time series with weekly resistance indexes (7D) calculated using the microbiology records available for the previous four weeks (4x7D). It is important to note that some notations are equivalent representations of the same susceptibility data at different granularity, hence their slopes are comparable. As an example, the trend estimated for $1 \mathrm{M}_{1}$ should be approximately thirty times the trend estimated for $1 \mathrm{D}_{30}$. 
Table 1. Description of strategies to generate resistance time series.

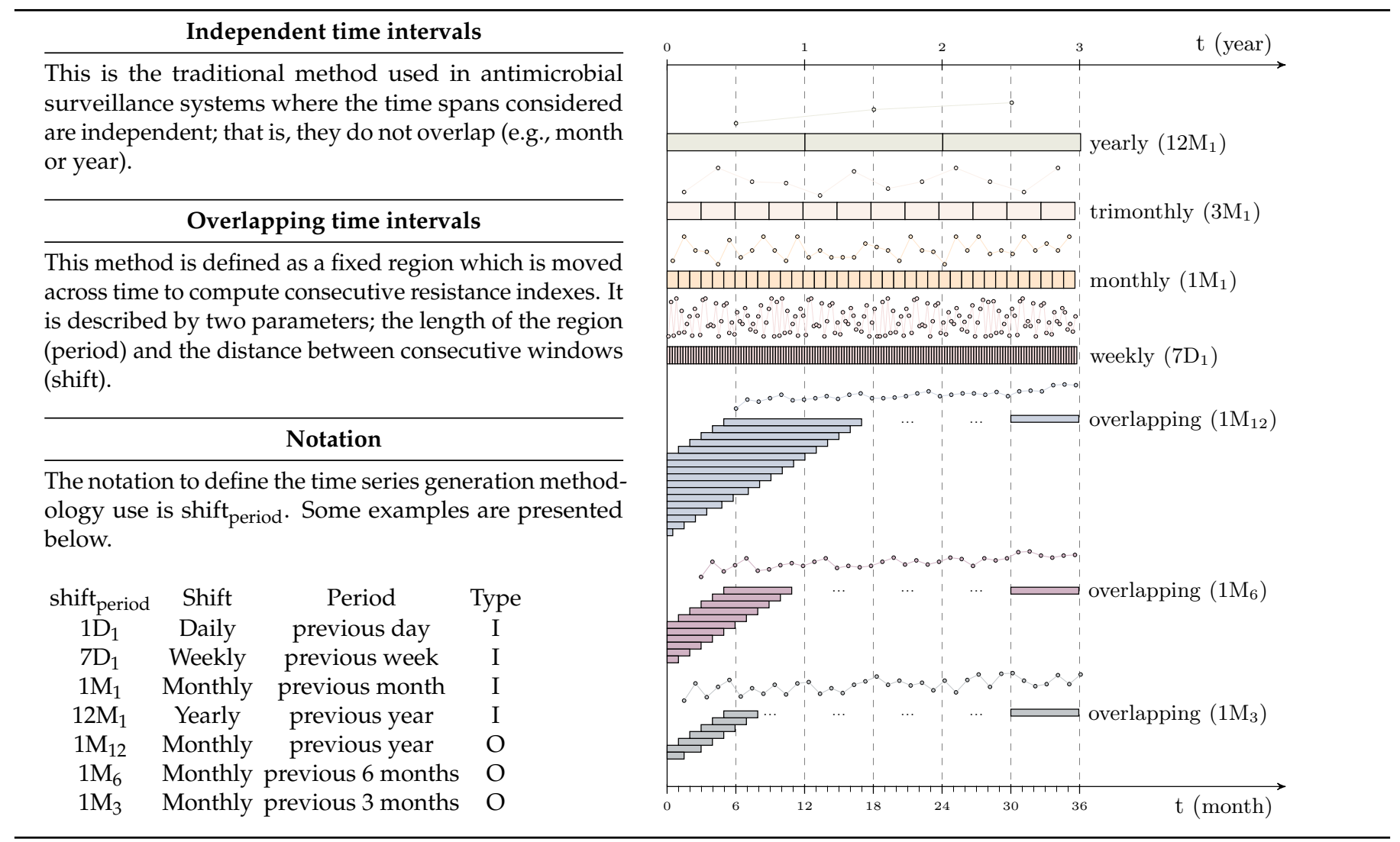

Keys: $D=$ day; $M=$ month; $I=$ independent time intervals; $O=$ overlapping time intervals.

\subsection{Regression Analysis for Trend Estimation}

The linear model (see Equation (2)) has been selected to quantify resistance tendency for several reasons: (i) the development of resistance in pathogens is an evolutionary response hence large variations in short periods (e.g., consecutive days or months) are not expected; (ii) the slope parameter can be directly translated to change over time increasing its practicability; and (iii) the offset parameter is highly related with the overall resistance. Hence, the response variable in regression analysis (resistance index) is described by the explanatory variable (time). The slope $(m)$ ranges within the interval $[-1,1]$ where sign and absolute value capture direction and rate of change respectively. The unit of the slope is represented by $\Delta_{y} / \Delta_{x}$. It has been denoted as Single Antimicrobial Resistance Trend (SART).

$$
y=m x+n \quad \text { where } \quad m=\frac{y_{t+1}-y_{t}}{x_{t+1}-x_{t}} .
$$

\subsubsection{Least Squares Regression}

The optimization problem in ordinary least squares (OLS) regression minimizes the least square errors to find the best fitting model as described in Equation (3). These errors $\left(\epsilon_{i}\right)$ are often called residuals and represent the differences between observed $(y)$ and estimated $\left(y^{\prime}\right)$ variables. Ordinary least squares assumes identical weights $\left(w_{i}\right)$ and independently distributed residuals with a normal distribution.

$$
\min _{m, n} \sum_{i=1}^{T} w_{i}^{2} \epsilon_{i}^{2} \text { where } \epsilon_{i}=y_{i}-y_{i}^{\prime}=y i-\left(m x_{i}+n\right) .
$$

It is frequently observed that some residuals might have higher variance than others, meaning that those observations are effectively less certain. To contemplate such variability, 
weighted linear squares (WLS) regression (see Equation (3)) applies a weighting function to the residuals. In this paper, the confidence of the computed resistance index (observed variable) relies on the number of susceptibility test records manipulated. Hence, the sigmoid function has been used to define weights proportional to the population size.

\subsubsection{Autoregressive Integrated Moving Average}

An autoregressive integrated moving average (ARIMA) model is a generalization of an autoregressive moving average (ARMA) model which can be also applied in scenarios where data show evidence of non-stationarity. The autoregressive (AR) part expresses the variable of interest (resistance index) as a function of past values of the variable. The moving average (MA) indicates that the regression error is a linear combination of error terms which occurred contemporaneously and at various times in the past. An ARIMA(p,d,q) model is defined as shown in Equation (4), where $p$ is the number of autoregressive terms, $\mathrm{d}$ is the number of differences needed for stationarity, $\mathrm{q}$ is the number of lagged forecast errors, and $\phi$ and $\theta$ are the coefficients of the model.

$$
y_{t}^{\prime}=\mu+\sum_{i=1}^{p} \phi_{i} y_{t-i}-\sum_{j=1}^{q} \theta_{j} y_{t-j} .
$$

The interpretation of the parameter $\mu$ depends on the ARIMA model used for the fitting. In order to estimate the linear trend, it was interesting to consider exclusively MA models so that the expected value of $\mu$ was the mean of the one-time differenced series; that is, the slope coefficient of the un-differenced series. The Bayesian information criterion (BIC) was used to select the best ARIMA $(0,1, q)$ model, being the one with the lowest BIC the preferred.

\subsection{Statistical Analysis}

\subsubsection{Trend and Stationarity in Time Series}

An analysis of stationarity around a trend was carried out to identify time series satisfying the assumptions posed by ARIMA. The augmented Dickey-Fuller test (ADF) was used to determine the presence of a unit root. When the other roots of the characteristic function lie inside the unit circle the first difference of the process is stationary. Due to this property, these are also called difference-stationary processes. Since the absence of unit root is not a proof of non-stationarity, the Kwiatkowski-Phillips-Schmidt-Shin (KPSS) test was used to identify the existence of an underlying trend which can also be removed to obtain a stationary process. These are called trend-stationary processes. In both unit-root and trend-stationary processes, the mean can increase or decrease over time; however, in the presence of a shock, trend-stationary processes revert to this mean tendency in the long run (deterministic trend) while unit-root processes have a permanent impact (stochastic trend). The significance level of the tests was set to 0.05 .

\subsubsection{Statistical Significance among Regression Methods}

The statistical significance of the differences among the regression methods was determined using the non parametric test Wilcoxon-Mann-Whitney (also denoted MannWhitney U) where the significance level was set to 0.05 .

\subsubsection{Pearson Correlation Coefficient}

It measures the linear correlation between two variables with a value within the range $[-1,1]$. Coefficient values of $-1,0$ and 1 indicate total negative linear correlation, no linear correlation and total positive correlation respectively. In this study, the coefficient is used to assess whether or not there is a linear correlation between the number of observations (susceptibility test records) and the computed resistance index. 


\subsection{Software}

The Python programming language was used in this research. The libraries used for time series analysis and data handling were Statsmodels [35] and Pandas [36] respectively. Additionally, Matplotlib [37] and Seaborn [38] were used for data visualization.

\section{Results}

\subsection{Analysis of the Robustness of the Methods}

The process to generate a resistance time series signal from susceptibility data is defined by two parameters: shift and period. This section compares the robustness of three regression analysis methods (OLS, WLS and ARIMA) to quantify secular trends for resistance time series generated using different parameter configurations. For such purpose, the absolute difference between paired trends (SART distances) has been computed. The distribution of such distances is shown in Figure 2 for consecutive periods (left) and various granularities (right). Lower values indicate higher consistency in the estimation of trends.
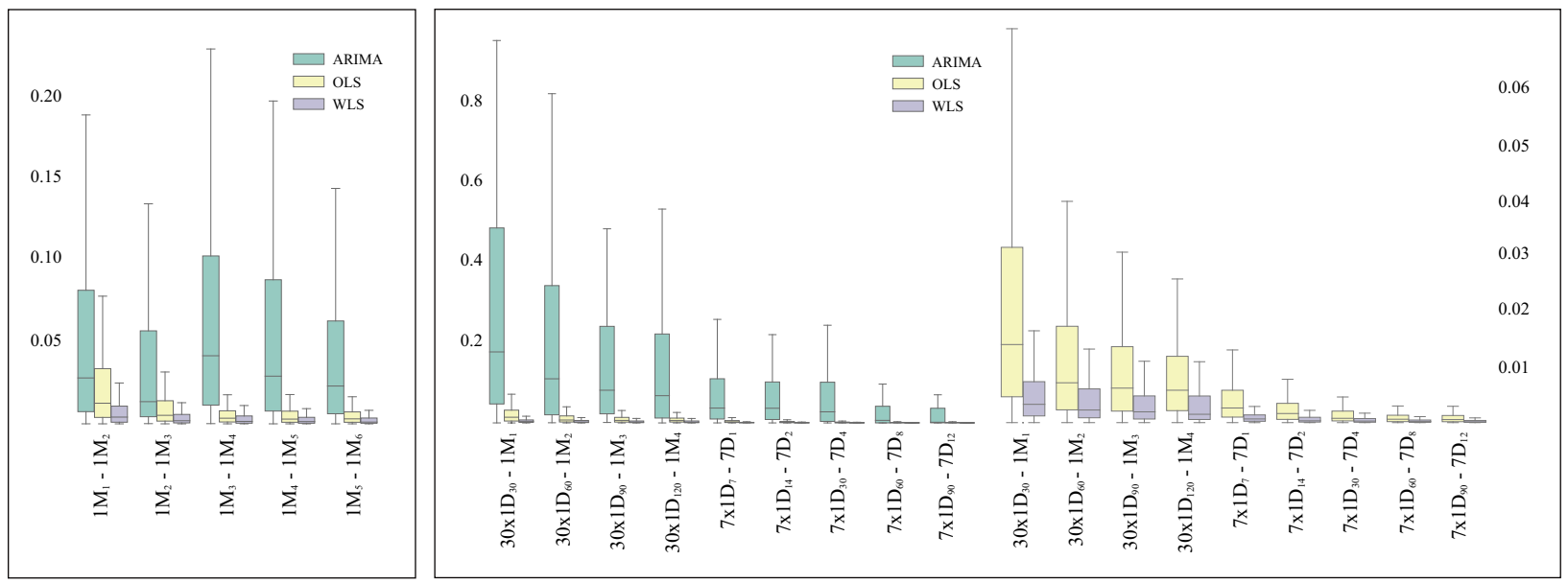

Figure 2. Distribution of paired SART distances. Comparison of ordinary least squares (OLS), weighted least squares (WLS) and autoregressive integrated moving average (ARIMA) in the following scenarios: consecutive periods (left) and equivalent granularities (right). An additional graph including exclusively OLS and WLS has been added on the latter to facilitate their comparison. The x-axis represents couplets of configurations to generate resistance time series under comparison.

\subsubsection{Consistency on Consecutive Time Spans}

The length of the period determines the amount of susceptibility test records accounted to compute the resistance index. Lengthy periods provide smoother time series which are better approximated by the linear model, especially when overlapping time periods are considered. As a consequence, the SART distances decrease as shown by the median of the distributions (see left graph in Figure 2). This behaviour is consistent in OLS and WLS. However, it is worth highlighting the irregularities shown by ARIMA. The median and quartiles of the distributions indicate that WLS produces the most stable results and it is followed closely by OLS. Nonetheless, there is a considerable gap between these two methods and ARIMA. All the distances estimated by WLS were significantly smaller $(p<0.001)$ than those obtained using OLS and ARIMA.

\subsubsection{Consistency on Granularity}

The SART measures ratio of change per time unit. Therefore, the monthly trend should be approximately four times the weekly trend and thirty times the daily trend. These correspondences are shown in Figure 2. Firstly, it is important to notice the substantial variation in the distribution of SART distances, which is one order of magnitude larger for ARIMA. Consequently, ARIMA has not been further considered for trend estimation. For the sake of clarity, the distribution of SART distances for OLS and WLS have been 
represented separately (see right graph in Figure 2). WLS presents the best performance in terms of granularity and the disparity with OLS is particularly visible for those scenarios in which independent time periods are used $\left(1 \mathrm{M}_{1}\right.$ and $\left.7 \mathrm{D}_{1}\right)$. All the distances estimated by WLS were significantly smaller $(p<0.001)$ than those obtained using OLS and ARIMA.

\subsection{AMR Surveillance: Case Studies}

The most commonly requested sample types were urine $(30 \%)$, wound $(26 \%)$ and blood $(6 \%)$ with the majority of tests corresponding to a reduced set of pathogens which are a common cause of infection. The most representative pathogens were Escherichia coli (51\% in urine cultures and $26 \%$ in blood cultures) and Staphylococcus aureus (49\% in wound cultures). As such, to provide a detailed insight and validate the estimated resistance rates and trends, three case studies are presented below: (i) Escherichia coli in blood samples; (ii) Escherichia coli in urine samples; and (iii) Staphylococcus aureus in wound samples.

The presented case studies (see Tables 2-4, Figures 3-5) report the resistance rates and resistance trends (monthly and yearly) with the corresponding confidence intervals for various antimicrobials. These results are supported with references to the existing literature. The last two columns present the Pearson correlation coefficient and the total number of isolates. For the sake of clarity, the resistance rate, the resistance trend and the Pearson correlation coefficient have been displayed graphically. In addition, a number of resistance time series have been represented graphically (see Tables 2-4, Figures 3-5) including the number of susceptibility tests (bars), the corresponding resistance index (circle) and the estimated linear trend (overlay straight line).

Table 2. AMR summary for E. coli in urine samples.

\begin{tabular}{|c|c|c|c|c|c|c|c|}
\hline Antimicrobial & $\mathrm{R}(\%)(95 \% \mathrm{CI})$ & References & $T_{M}(\%)(95 \% C I)$ & References & $T_{Y}(\%)$ & Pearson & Isolates \\
\hline Cephalexin (CELX) & $\mathbf{1 1 . 1}(10.9,11.3)$ & & $\mathbf{0 . 0 5 5}(0.045,0.065)$ & & $0.7 \uparrow$ & -0.25 & 79,090 \\
\hline Ciprofloxacin (CIP) & $16.3(16.0,16.5)$ & {$[39,40]$} & $\mathbf{0 . 0 4 6}(0.031,0.062)$ & {$[5,40]$} & $0.6 \uparrow$ & -0.46 & 79,239 \\
\hline Trimethoprim (TRI) & $37.8(37.4,38.1)$ & [39-42] & $0.033(0.020,0.046)$ & [40] & $0.4 \uparrow$ & -0.14 & 79,133 \\
\hline Augmentin (AUG) & $10.9(10.7,11.2)$ & & $\mathbf{0 . 0 1 8}(-0.022,0.059)$ & & $0.2 \leftrightarrow$ & -0.42 & 79,093 \\
\hline Meropenem (MER) & $0.2(0.1,0.3)$ & & $\mathbf{0 . 0 0 2}(-0.002,0.006)$ & & $0.0 \leftrightarrow$ & 0.02 & 9875 \\
\hline Nitrofurantoin (NIT) & $2.7(2.6,2.8)$ & [39-42] & $-0.006(-0.013,0.001)$ & & $-0.1 \leftrightarrow$ & -0.18 & 79,108 \\
\hline Amikacin (AMI) & $1.1(0.9,1.2)$ & & $-0.011(-0.022,0.000)$ & & $-0.1 \leftrightarrow$ & -0.23 & 9786 \\
\hline Cefotaxime (CTX) & $60.8(59.9,61.8)$ & & $-\mathbf{0 . 0 1 2}(-0.083,0.059)$ & & $-0.1 \leftrightarrow$ & 0.01 & 9803 \\
\hline Tazocin (TAZ) & $24.2(23.3,25.0)$ & [39] & $-\mathbf{0 . 0 2 3}(-0.078,0.032)$ & & $-0.3 \leftrightarrow$ & 0.01 & 9878 \\
\hline Gentamicin (GEN) & $9.3(9.1,9.5)$ & {$[42]$} & $-\mathbf{0 . 0 3 3}(-0.061,-0.005)$ & & $-0.4 \downarrow$ & -0.62 & 63,399 \\
\hline Ertapenem (ERT) & $2.0(1.7,2.3)$ & & $-\mathbf{0 . 0 3 3}(-0.050,-0.017)$ & & $-0.4 \downarrow$ & -0.31 & 8882 \\
\hline Ceftazidime (CAZ) & $57.3(53.3,58.2)$ & & $-\mathbf{0 . 0 3 8}(-0.113,0.037)$ & & $-0.5 \leftrightarrow$ & -0.04 & 9810 \\
\hline Mecillinam (MEC) & $5.4(4.9,5.8)$ & & $-0.048(-0.071,-0.024)$ & & $-0.6 \downarrow$ & -0.29 & 9083 \\
\hline Cefoxitin (CXT) & $26.0(25.1,26.8)$ & & $-0.069(-0.123,-0.016)$ & & $-0.8 \downarrow$ & 0.15 & 9798 \\
\hline
\end{tabular}

Keys: $\mathrm{CI}=$ confidence interval; $\mathrm{R}=$ resistance; $\mathrm{T}_{\mathrm{M}}=$ monthly trend; $\mathrm{T}_{\mathrm{Y}}=$ yearly trend; $\uparrow=$ significant increase; $\downarrow=$ significant decrease. Significance: A trend is significant if the CI does not include 0 .
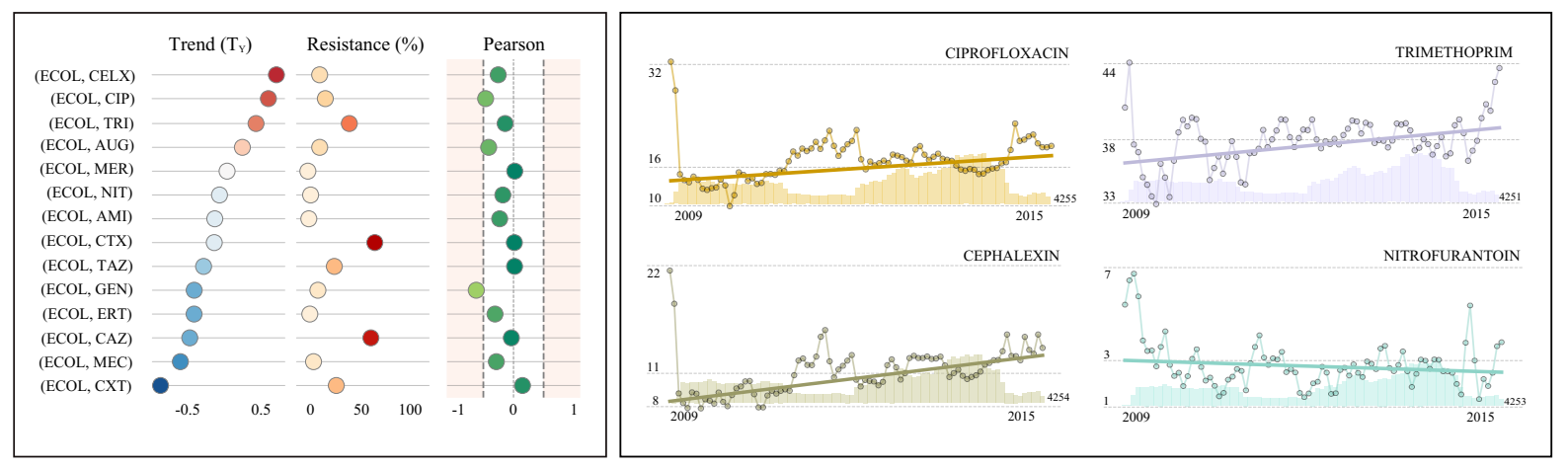

Figure 3. Case study I: E. coli in urine samples. The table presents the resistance index, resistance trend (monthly and yearly), the pearson correlation coefficient, the number of isolates and external resources for validation for each antimicrobial. In addition, the main metrics (left) and four examples of resistance time series (right) are graphically represented to facilitate comparison. 
Table 3. AMR summary for E. coli in blood samples.

\begin{tabular}{|c|c|c|c|c|c|c|c|}
\hline Antimicrobial & $R(\%)(95 \% \mathrm{CI})$ & References & $\mathrm{T}_{\mathrm{M}}(\%)(95 \% \mathrm{CI})$ & References & $T_{Y}(\%)$ & Pearson & Isolates \\
\hline Augmentin (AUG) & $47.5(45.8-49.2)$ & {$[5,41]$} & $0.359(0.249,0.470)$ & [41] & $4.3 \uparrow$ & 0.64 & 3317 \\
\hline Trimethoprim (TRI) & $47.2(45.4-49.1)$ & & $\mathbf{0 . 1 9 0}(0.079,0.301)$ & & $2.3 \uparrow$ & 0.01 & 2774 \\
\hline Cefoxitin (CXT) & $11.5(10.4-12.6)$ & & $\mathbf{0 . 0 4 1}(-0.006,0.089)$ & & $0.5 \leftrightarrow$ & 0.22 & 3316 \\
\hline Tazocin (TAZ) & $13.7(12.6-14.9)$ & {$[5,41,43]$} & $0.006(-0.040,0.052)$ & {$[5,41,43]$} & $0.1 \leftrightarrow$ & -0.08 & 3321 \\
\hline Tigecycline (TIG) & $1.7(1.2-2.2)$ & & $\mathbf{0 . 0 0 2}(-0.026,0.030)$ & & $0.0 \leftrightarrow$ & 0.45 & 2734 \\
\hline Gentamicin (GEN) & $16.6(15.3-17.8)$ & {$[5,44]$} & $\mathbf{0 . 0 0 0}(-0.044,0.045)$ & {$[5,44]$} & $0.0 \leftrightarrow$ & -0.12 & 3322 \\
\hline Meropenem (MER) & $0.5(0.3-0.8)$ & {$[5,41,43,44]$} & $-0.001(-0.020,0.018)$ & {$[5,41,43,44]$} & $0.0 \leftrightarrow$ & -0.05 & 3280 \\
\hline Temocillin (TEM) & $11.1(10.0-12.2)$ & & $-0.002(-0.086,0.082)$ & & $0.0 \leftrightarrow$ & 0.42 & 3044 \\
\hline Ertapenem (ERT) & $1.2(0.8-1.6)$ & & $-0.005(-0.025,0.016)$ & & $-0.1 \leftrightarrow$ & -0.28 & 2992 \\
\hline Aztreonam (AZT) & $19.6(18.1-21.0)$ & & $-\mathbf{0 . 0 1 2}(-0.077,0.052)$ & & $-0.1 \leftrightarrow$ & -0.26 & 2925 \\
\hline Amoxicillin (AMO) & $72.7(71.2-74.2)$ & & $-0.017(-0.085,0.051)$ & & $-0.2 \leftrightarrow$ & -0.06 & 3319 \\
\hline Amikacin (AMI) & $1.6(1.2-2.1)$ & & $-0.018(-0.041,0.006)$ & & $-0.2 \leftrightarrow$ & -0.23 & 3044 \\
\hline Ceftazidime (CAZ) & $19.3(17.9-20.6)$ & {$[27,44]$} & $-\mathbf{0 . 0 1 9}(-0.065,0.027)$ & [27] & $-0.2 \leftrightarrow$ & -0.33 & 3323 \\
\hline Cefotaxime (CTX) & $20.3(18.9-21.7)$ & {$[27,44]$} & $-0.021(-0.070,0.027)$ & {$[27]$} & $-0.3 \leftrightarrow$ & -0.31 & 3201 \\
\hline Ciprofloxacin (CIP) & $35.2(33.6-36.8)$ & [44] & $-\mathbf{0 . 0 3 5}(-0.017,0.037)$ & & $-0.4 \leftrightarrow$ & -0.35 & 3320 \\
\hline Cefuroxime (CXM) & $24.2(22.8-25.7)$ & & $-\mathbf{0 . 0 8 0}(-0.137,-0.024)$ & & $-1.0 \downarrow$ & -0.39 & 3320 \\
\hline Tobramycin (TOB) & $22.1(20.6-23.6)$ & & $-0.099(-0.188,-0.010)$ & & $-1.2 \downarrow$ & -0.65 & 2832 \\
\hline Colistin (COL) & $4.0(3.3-4.8)$ & & $-0.208(-0.274,-0.141)$ & & $-2.5 \downarrow$ & -0.37 & 2606 \\
\hline
\end{tabular}

Keys: $\mathrm{CI}=$ confidence interval; $\mathrm{R}=$ resistance; $\mathrm{T}_{\mathrm{M}}=$ monthly trend; $\mathrm{T}_{\mathrm{Y}}=$ yearly trend; $\uparrow=$ significant increase; $\downarrow=$ significant decrease.

Significance: A trend is significant if the CI does not include 0 .
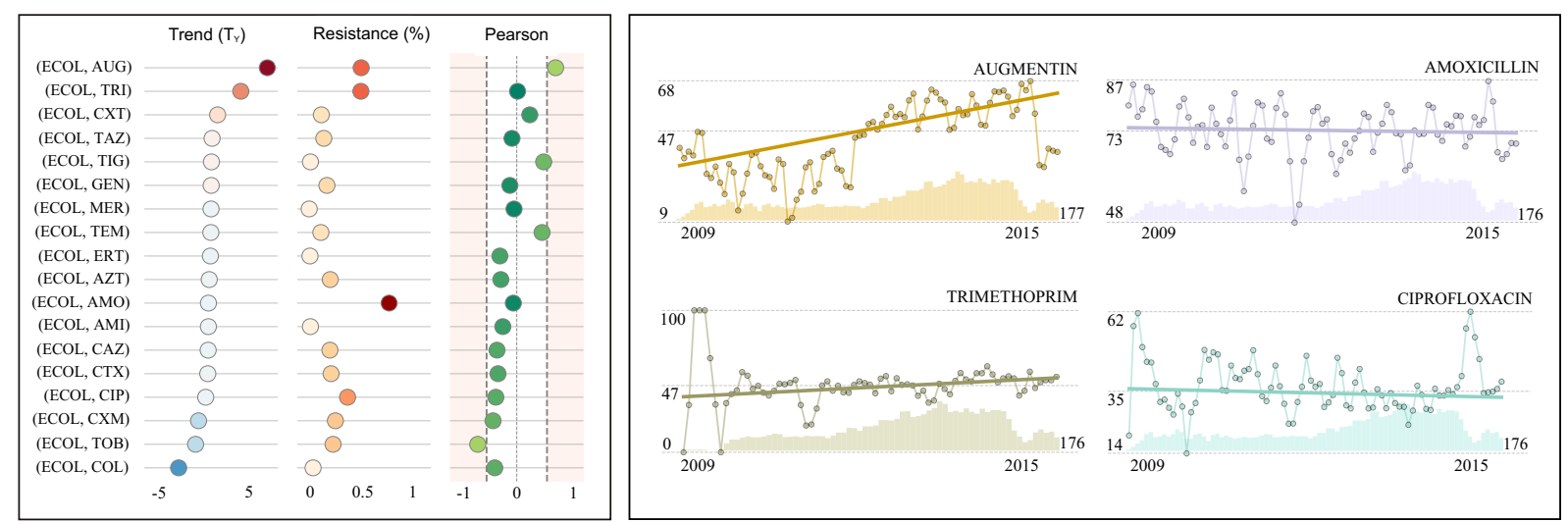

Figure 4. Case study II: E. coli in blood samples. The table presents the resistance index, resistance trend (monthly and yearly), the pearson correlation coefficient, the number of isolates and external resources for validation for each antimicrobial. In addition, the main metrics (left) and four examples of resistance time series (right) are graphically represented to facilitate comparison.

Table 4. AMR summary for S. aureus in wound samples.

\begin{tabular}{|c|c|c|c|c|c|c|c|}
\hline Antimicrobial & $R(\%)(95 \% C I)$ & References & $\mathrm{T}_{\mathrm{M}}(\%)(95 \% \mathrm{CI})$ & References & $\mathrm{T}_{\mathrm{Y}}(\%)$ & Pearson & Isolates \\
\hline Trimethoprim (TRI) & $10.1(9.8,10.4)$ & & $0.052(0.026,0.077)$ & & $0.6 \uparrow$ & 0.10 & 33,525 \\
\hline Penicillin (PEN) & $89.4(89.1,89.7)$ & & $\mathbf{0 . 0 5 0}(0.034,0.065)$ & & $0.6 \uparrow$ & 0.19 & 39,901 \\
\hline Rifampicin (RIF) & $1.7(1.5,1.8)$ & [45] & $\mathbf{0 . 0 0 1}(-0.015,0.017)$ & [45] & $0.0 \leftrightarrow$ & 0.23 & 35,141 \\
\hline Mupirocin (MUP) & $2.5(2.3,2.6)$ & & $-0.001(-0.020,0.017)$ & & $0.0 \leftrightarrow$ & -0.39 & 33,716 \\
\hline Gentamicin (GEN) & $4.1(3.9,4.3)$ & & $-0.003(-0.023,0.018)$ & & $0.0 \leftrightarrow$ & -0.16 & 35,255 \\
\hline Clindamycin (CLI) & $22.4(22.0,22.8)$ & & $-0.016(-0.034,0.001)$ & & $-0.2 \leftrightarrow$ & -0.24 & 39,962 \\
\hline Tetracycline (TET) & $9.7(9.4,10.0)$ & & $-\mathbf{0 . 0 1 8}(-0.041,0.004)$ & & $-0.2 \leftrightarrow$ & 0.15 & 35,429 \\
\hline Fusidic acid (FUS) & $14.5(14.2,14.9)$ & & $-0.025(-0.044,-0.006$ & & $-0.3 \downarrow$ & 0.04 & 39,918 \\
\hline Erythromicin (ERY) & $26.0(25.6,26.5)$ & & $-\mathbf{0 . 0 3 2}(-0.049,-0.015$ & & $-0.4 \downarrow$ & -0.24 & 39,971 \\
\hline Meticillin (MET) & $15.3(14.9,15.7)$ & [41] & $-0.090(-0.113,-0.068$ & [41] & $-1.1 \downarrow$ & -0.45 & 39,950 \\
\hline Ciprofloxacin (CIP) & $20.1(19.7,20.5)$ & & $-0.116(-0.156,-0.075$ & & $-1.4 \downarrow$ & -0.62 & 35,227 \\
\hline
\end{tabular}

Keys: $\mathrm{CI}=$ confidence interval; $\mathrm{R}=$ resistance; $\mathrm{T}_{\mathrm{M}}=$ monthly trend; $\mathrm{T}_{\mathrm{Y}}=$ yearly trend; $\uparrow=$ significant increase; $\downarrow=$ significant decrease. Significance: A trend is significant if the CI does not include 0 . 

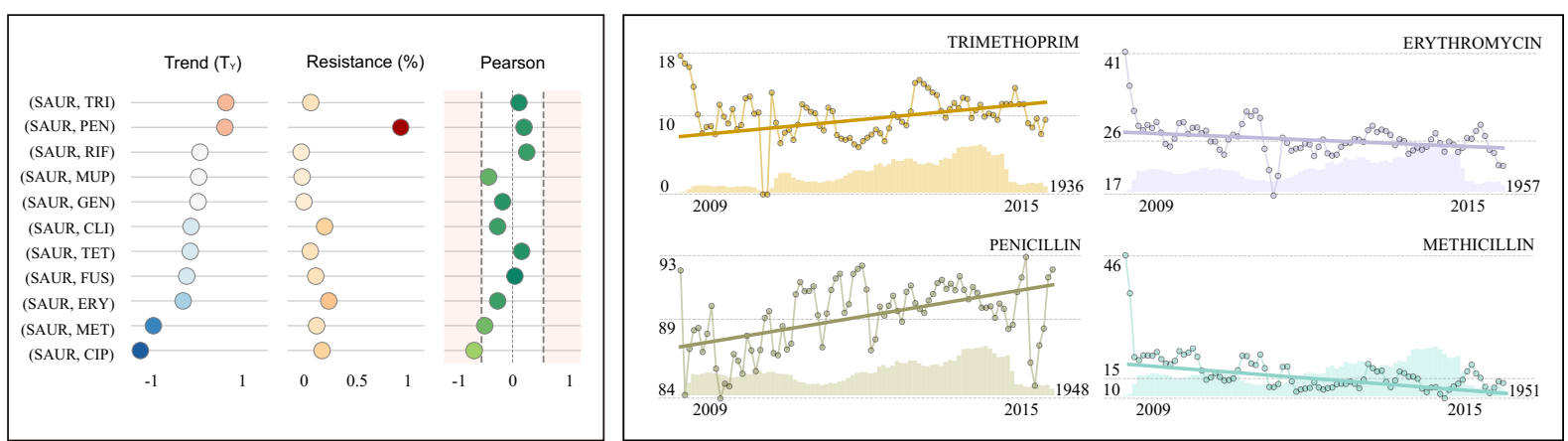

Figure 5. Case study III: S.aureus in wound samples. The table presents the resistance index, resistance trend (monthly and yearly), the Pearson correlation coefficient, the number of isolates and external resources for validation for each antimicrobial. In addition, the main metrics (left) and four examples of resistance time series (right) are graphically represented to facilitate comparison.

\section{Discussion}

The process to generate a resistance time series signal from susceptibility data is defined by two parameters: shift and period. Regardless of the value of these parameters, the estimated trends should be independent of the granularity (shift) and show a consistent change when time spans overlap (period). OLS is perhaps the most popular method for trend estimation and has shown consistent results in our study. However, it is known to be greatly affected by outliers. To palliate this effect, WLS has been considered to reduce the contribution of outliers by considering the number of susceptibility tests available. While ARIMA is a very popular suite of models which has proven to be robust in short-term forecasting, it has two main limitations: (i)requires stationary time series and (ii) parameter tuning is not straightforward. Altogether, WLS was selected as the preferred method for trend estimation since it was robust against changes in the granularity of the time series and presented the best performance. In addition, it is easy to comprehend and use increasing its practicability and implementation in other institutions.

\subsection{Case Study I: Escherichia coli in Urine Samples}

Escherichia coli (ECOL) is a gram negative bacteria and most strains are harmless, being part of the normal flora of the gut. However, virulent strains can cause gastroenteritis, urinary tract infection, meningitis and Crohn's disease. It is the most widely studied pathogen since it is easy to reproduce under favourable conditions. E. coli is responsible for more than $85 \%$ of all urinary tract infections. There is an alarming resistance to cefotaxime $(60.8 \%)$, ceftazidime (57.3\%) and trimethoprim $(37.8 \%)$ with equivalent results in other studies (see table in Figure 3). Furthermore, there has been a noticeable increase in resistance to cephalexin $(0.7 \%)$, ciprofloxacin $(0.6 \%)$ and trimethoprim $(0.4 \%)$. On the other side, resistance to Augmentin (0.2\%) is positive yet not significant since the confidence intervals contain 0 . Nitrofurantoin is commonly identified as one of the most active agents to treat $E$. coli with resistance rates within the range 3.7-6\% in 2003-2008 [39,42], further stabilized to $3 \%$ in 2015-2017 [5,41]. These rates harmonize with those presented in the corresponding resistance time series (see time series graph for nitrofurantoin in Figure 3) and the estimated marginally decreasing trend $(-0.01 \%)$. While the resistance rate to nitrofurantoin $(2.7 \%)$ is low, there are antimicrobials with even lower rates such as ertapenem $(2.0 \%)$ or amikacin (1.1\%). Furthermore, carbapenems show negligible resistance rates; meropenem $(0.2 \%)$ and imipenem $(0.2 \%)$.

\subsection{Case Study II: Escherichia coli in Blood Samples}

The national mandatory surveillance program has reported a consistent rise in the incidences of E. coli bacteremia in England [5]. Furthermore, the majority of antimicrobials under surveillance also presented an increase in resistance rates over the last years (see Figure 4). Such rising resistance rates are particularly significant for Augmentin (4.3\%) and 
trimethoprim (2.3\%). Note that, although the resistance trend for Augmentin is significant, the pearson coefficient indicates a high correlation between the number of records and the estimated resistance index. The introduction of MALDI-TOF mass spectrometry in 2011 might have caused this effect (see time series graph for Augmentin in Figure 4). The high proportion of resistant isolates presented for these two antimicrobials $(47.5 \%$ and $47.2 \%$ respectively) is only overpassed by amoxicillin $(72.7 \%)$. Thus, there should be concerns on the use of these antimicrobials in clinical practice. Ciprofloxacin presents the fourth highest proportion of resistant isolates $(35.2 \%)$, which has been slightly decreasing in recent years [27]. On the contrary, surveillance in carbapenems shows negligible resistance rates which have remained constant over the years [5]. For instance, meropenem and ertapenem resistance rates $(0.5 \%$ and $1.2 \%)$ and trends $(0.0 \%$ and $-0.1 \%)$ are shown in Figure 4.

\subsection{Case Study III: Staphylococcus aureus in Wound Samples}

Staphylococcus aureus (SAUR) is a Gram-positive bacteria typically found in the respiratory tract and the skin. It is a leading cause of bloodstream infections [46,47], generally associated with breakages in the skin due to surgery, injury or use of intra-vascular devices such as catheters. Therefore, it is frequently acquired in hospitals [48]. Penicillin-resistant isolates were recognised in 1942 [49] reaching a proportion of $80 \%$ by late 1960 s. Nowadays, the resistance rate to penicillin $(89.4 \%)$ is the highest and has shown an increasing trend $(0.6 \%)$ over the last years. There was also an emergence of methicillin-resistant Staphylococcus aureus (MRSA). It was first reported in a British hospital and became a worldwide problem in clinical medicine [27] with a peak of 43\% in 2001 [50]. The Department of Health in England made reduction in rates of MRSA a priority with improvement of surveillance as one of their first actions. This led to a decrease in the number of resistant cases reported [51]. Such decrease continued in 2012-2015 [41,52] and coincides with the negative trend $(-1.1 \%)$ presented in Figure 5 and the corresponding time series graph. Nowadays, approximately $15.3 \%$ of isolates are methicillin-resistant [41]. Also, there should be concerns on antimicrobials such as erythromycin $(26.0 \%)$ and clindamycin $(22.4 \%)$ with higher resistance rates and no further evidence of improvement. Moreover, while resistance rates to trimethoprim are not very high $(10.1 \%)$, it has presented a noticeable rise $(0.6 \%)$ in the last years. Since trimethoprim is clinically valuable to treat skin and soft tissue infections caused by MRSA, such rise constitutes a major threat. On the other side, rifampicin-containing treatments are known to improve outcomes in Staphylococcal wound infections presenting the lowest resistance rate $(1.7 \%)$ and a constant trend $(0.0 \%)[45]$.

\subsection{Susceptibility Testing: Behaviour and Guidelines}

The information extracted from antimicrobial surveillance is valuable to guide and support antimicrobial therapy selection. However, the reliability of this information highly depends on the number of observations available which might vary considerably (see isolates in Figure 3). For instance, a large proportion of combinations had an insufficient number of susceptibility tests to perform resistance trend estimation. The disparity among the pathogens tested is induced by the hospital occurrence rate; leading to higher number of tests for pathogens which are a common cause of infection in the population. On the other side, laboratory guidelines promote susceptibility testing for a limited range of antimicrobial agents that, based on pharmacological and empirical knowledge, may potentially be able to inhibit or kill the pathogen. This selection, generally based on national laboratory guidelines, causes the corresponding disparity among antimicrobials. Note that these guidelines might not be appropriate for the needs of each hospital. Thus, developing effective communication strategies, such as the resistance summaries presented in this work, could provide the necessary knowledge to revise and potentially tailor them accordingly.

\subsection{Advantages of Overlapping Time Intervals in Surveillance}

Antimicrobial surveillance is performed at different levels (e.g., local or national) and it is greatly affected by the size of the dataset considered. Versatile yet efficient analytic 
methods are required in those scenarios where data access or availability is restricted, such as clinical research. The main advantages of overlapping time intervals are: (i) it is a flexible approach which enables to adjust granularity and accuracy (ii) resulting time series are visually more legible and insightful (iii) enables the study of short-time variations in contrast to current mechanisms using sparse data points (years apart) and (iv) the outcomes are more consistent. On the other side, it might originate certain relationship between consecutive observations as data is partially shared. Overall, this step is optional and might be particularly useful in scenarios where data is limited but decent levels of granularity are still required.

\subsection{The Importance of Surveillance Data}

Despite global antimicrobial surveillance becoming a priority in recent years, homogeneity of antimicrobial policies does still produce different antimicrobial resistance outcomes [8]. For instance, it is widely documented that resistance rates are considerably higher in London than in the rest of UK, emphasizing the significance of local AMR surveillance. Health care organizations benefit from data on rates of antimicrobial resistance in many ways: (i) contributes to the evidence base used for formulation of national treatment guidelines (ii) can be used to assess the effectiveness and impact of interventions and (iii) has a key role in detecting the emergence and spread of previously uncommon or completely novel types of resistance. Furthermore, AMR surveillance plays a major role in patient management by providing data that influences clinical decision-making [9]. Since it guides antimicrobial selection for empirical treatment it is crucial at point of care. For such reason, this information will be integrated in Enhanced Personalized and Integrated Care for Infection Management at Point of Care (EPIC IMPOC), a modular intelligent decision support system which aims to assist clinicians at the different stages of the infection management pathway [53-57].

\subsection{Limitations}

The data considered in this study were not collected purposely. Therefore, it could be influenced by external factors such as changes in susceptibility testing policies (e.g., MIC breakpoints), technology or outbreaks. For instance, the introduction of MALDI-TOF mass spectrometry in 2011 might have affected the number of microbiology tests requested by clinicians. However, while testing relies on hospital policies, suspicion of infection was assumed for all microbiology tests requested.

\section{Conclusions}

Surveillance is the cornerstone for assessing the burden of antimicrobial resistance and strengthens knowledge for action in support of stewardship program strategies by improving existing guidelines. The efficient use of susceptibility data provided by the overlapping time spans drops the dependence between the granularity and accuracy of traditional surveillance systems. The robustness of weighted least squares regression facilitates resistance trend estimation and could be used to enhance existing surveillance systems which exclusively focus on resistant rates. Furthermore, there is an opportunity to investigate seasonal or other cyclic variations. Automating and facilitating access to surveillance reports through clinical decision support systems would enhance resistance awareness among clinicians and possibly have an impact on antimicrobial prescription practices.

Author Contributions: Conceptualization, B.H. and T.M.R.; methodology, B.H. and T.M.R.; software, B.H.; validation, T.M.R., L.S.P.M. and A.H.H.; formal analysis, B.H. and T.M.R.; investigation, B.H. and T.M.R.; resources, B.H. and T.M.R.; data curation, B.H.; writing-original draft preparation, B.H.; writing-review and editing, B.H., P.H.-V., T.M.R., L.S.P.M., P.G. and A.H.H.; visualization, B.H.; supervision, P.H.-V. and P.G.; project administration, B.H. and T.M.R.; funding acquisition, P.H.-V., P.G. and A.H.H. All authors have read and agreed to the published version of the manuscript. 
Funding: This research was funded by the National Institute for Health Research Invention for Innovation (i4i) grant, Enhanced, Personalized and Integrated Care for Infection Management at Point of Care (EPIC IMPOC), II-LA-0214-20008.

Institutional Review Board Statement: The study protocol was reviewed by the West London Regional Ethics Committee (REC) and deemed to not require ethics approval with monitoring under service evaluation governance structures being recommended by the REC as more appropriate for this study (REC 15/LO/1269 / ICHNT Service Evaluation SE113). Anonymised data was provided to the research team following local information governance procedures in line with this approval.

Informed Consent Statement: Not Applicable.

Data Availability Statement: The processed datasets analysed during the current study are available from the corresponding author (B.H.) on reasonable request, as long as this meets local ethics and research governance criteria.

Acknowledgments: The authors acknowledge the National Institute for Health Research Health Protection Research Unit (NIHR HPRU) in Healthcare Associated Infection and Antimicrobial Resistance at Imperial College London in partnership with Public Health England and the NIHR Imperial Patient Safety Translational Research Centre. The Department of Health and Social Care-funded Centre for Antimicrobial Optimization (CAMO), Imperial College London provides state-of-the-art research facilities and consolidates multidisciplinary academic excellence, clinical expertise, Imperial's NIHR/Wellcome-funded Clinical Research Facility (CRF) and partnerships with the National Health Service (NHS) to support and deliver innovative research on antimicrobial optimization and precision prescribing. The views expressed in this publication are those of the authors and not necessarily those of the NHS, the NIHR or the UK Department of Health.

Conflicts of Interest: A.H.H. and L.S.P.M. consulted for bioMerieux in 2013 and 2014 respectively. All other authors have no conflicts of interest to declare.

\section{Abbreviations}

The following abbreviations are used in this manuscript:

$\begin{array}{ll}\text { ADF } & \text { Augmented Dickey-Fuller } \\ \text { AMR } & \text { Antimicrobial Resistance } \\ \text { AMS } & \text { Antimicrobial Stewardship } \\ \text { AR } & \text { Autoregressive } \\ \text { ARIMA } & \text { Autoregressive Integrated Moving Average } \\ \text { ARMA } & \text { Autoregressive Moving-Average } \\ \text { BIC } & \text { Bayesian Information Criterion } \\ \text { CLSI } & \text { Clinical and Laboratory Standards Institute } \\ \text { ECOL } & \text { Escherichia coli } \\ \text { EUCAST } & \text { European Committee on Antimicrobial Susceptibility Testing } \\ \text { KPSS } & \text { Kwiatkowski-Phillips-Schmidt-Shin } \\ \text { MA } & \text { Moving Average } \\ \text { MALDI-TOF } & \text { Matrix Assisted Laser Desorption/Ionization-Time Of Flight } \\ \text { MARI } & \text { Multiple Antimicrobial Resistance Index } \\ \text { MIC } & \text { Minimum Inhibitory Concentration } \\ \text { MRSA } & \text { Methicillin Resistant Staphylococcus aureus } \\ \text { NHS } & \text { National Health Service } \\ \text { OLS } & \text { Ordinary Least Squares } \\ \text { SARI } & \text { Single Antimicrobial Resistance Index } \\ \text { SARIMA } & \text { Seasonal Autoregressive Integrated Moving Average } \\ \text { SART } & \text { Single Antimicrobial Resistance Trend } \\ \text { SAUR } & \text { Staphylococcus aureus } \\ \text { VARIMA } & \text { Vector Autoregressive Integrated Moving Average } \\ \text { WLS } & \text { Weighted Least Squares }\end{array}$




\section{References}

1. O'Neill, J. Antimicrobial resistance: Tackling a crisis for the health and wealth of nations. Rev. Antimicrob. Resist. 2014, $20,1-16$.

2. Wong, A. Epistasis and the evolution of antimicrobial resistance. Front. Microbiol. 2017, 8, 246. [CrossRef] [PubMed]

3. Holmes, A.H.; Moore, L.S.; Sundsfjord, A.; Steinbakk, M.; Regmi, S.; Karkey, A.; Guerin, P.J.; Piddock, L.J. Understanding the mechanisms and drivers of antimicrobial resistance. Lancet 2016, 387, 176-187. [CrossRef]

4. Collignon, P.; Athukorala, P.c.; Senanayake, S.; Khan, F. Antimicrobial resistance: The major contribution of poor governance and corruption to this growing problem. PLoS ONE 2015, 10, e0116746. [CrossRef] [PubMed]

5. Public Health England. English Surveillance Programme for Antimicrobial Utilisation and Resistance (ESPAUR); Annual Report 2017; PHE: London, UK, 2017.

6. European Centre for Disease Prevention and Control. Surveillance of Antimicrobial Resistance in Europe 2016; Annual report of the European Antimicrobial Resistance Surveillance Network (EARS-Net); ECDC: Stockholm, Sweden, 2017.

7. World Health Organization. Global Antimicrobial Resistance Surveillance System (GLASS) Report: Early Implementation 2016-2017; Licence: CC BY-NC-SA 3.0 IGO; WHO: Geneva, Switzerland, 2017.

8. Moore, L.S.P.; Freeman, R.; Gilchrist, M.J.; Gharbi, M.; Brannigan, E.T.; Donaldson, H.; Livermore, D.M.; Holmes, A.H. Homogeneity of antimicrobial policy, yet heterogeneity of antimicrobial resistance: Antimicrobial non susceptibility among 108717 clinical isolates from primary, secondary and tertiary care patients in London. J. Antimicrob. Chemother. 2014, 69, 3409. [CrossRef] [PubMed]

9. Rawson, T.M.; Wilson, R.C.; O’Hare, D.; Herrero, P.; Kambugu, A.; Lamorde, M.; Ellington, M.; Georgiou, P.; Cass, A.; Hope, W.W.; et al. Optimizing antimicrobial use: Challenges, advances and opportunities. Nat. Rev. Microbiol. 2021, 1-12.

10. Littmann, J.; Viens, A. The ethical significance of antimicrobial resistance. Public Health Ethics 2015, 8, 209-224. [CrossRef]

11. Pulcini, C.; Williams, F.; Molinari, N.; Davey, P.; Nathwani, D. Junior doctors' knowledge and perceptions of antibiotic resistance and prescribing: A survey in France and Scotland. Clin. Microbiol. Infect. 2011, 17, 80-87. [CrossRef]

12. Rodrigues, A.T.; Roque, F.; Falcão, A.; Figueiras, A.; Herdeiro, M.T. Understanding physician antibiotic prescribing behaviour: A systematic review of qualitative studies. Int. J. Antimicrob. Agents 2013, 41, 203-212. [CrossRef] [PubMed]

13. Doyle, M.P.; Loneragan, G.H.; Scott, H.M.; Singer, R.S. Antimicrobial resistance: Challenges and perspectives. Compr. Rev. Food Sci. Food Saf. 2013, 12, 234-248. [CrossRef]

14. Rawson, T.M.; Charani, E.; Moore, L.S.P.; Hernandez, B.; Castro-Sánchez, E.; Herrero, P.; Georgiou, P.; Holmes, A.H. Mapping the decision pathways of acute infection management in secondary care among UK medical physicians: A qualitative study. BMC Med. 2016, 14, 1-10. [CrossRef]

15. Charani, E.; Edwards, R.; Sevdalis, N.; Alexandrou, B.; Sibley, E.; Mullett, D.; Franklin, B.D.; Holmes, A. Behavior change strategies to influence antimicrobial prescribing in acute care: A systematic review. Clin. Infect. Dis. 2011, 53, 651-662. [CrossRef] [PubMed]

16. Davey, P.; Brown, E.; Charani, E.; Fenelon, L.; Gould, I.M.; Holmes, A.; Ramsay, C.R.; Wiffen, P.J.; Wilcox, M. Interventions to improve antibiotic prescribing practices for hospital inpatients. Cochrane Libr. 2017, 2, 003543. [CrossRef] [PubMed]

17. Pinder, R.; Berry, D.; Sallis, A.; Chadborn, T. Antibiotic Prescribing and Behaviour Change in Healthcare Settings: Literature Review and Behavioural Analysis; Department of Health \& Public Health England: London, UK, 2015.

18. Elias, C.; Moja, L.; Mertz, D.; Loeb, M.; Forte, G.; Magrini, N. Guideline recommendations and antimicrobial resistance: The need for a change. BMJ Open 2017, 7, e016264. [CrossRef] [PubMed]

19. Nair, S.; Hsu, D.; Celi, L.A. Challenges and Opportunities in Secondary Analyses of Electronic Health Record Data. In Secondary Analysis of Electronic Health Records; Springer International Publishing: Cham, Switzerland, 2016; pp. 17-26. [CrossRef]

20. Department of Health. UK Five Year Antimicrobial Resistance Strategy: 2013 to 2018; Department of Health: London, UK, 2013.

21. Goldmann, D.A.; Weinstein, R.A.; Wenzel, R.P.; Tablan, O.C.; Duma, R.J.; Gaynes, R.P.; Schlosser, J.; Martone, W.J.; Acar, J.; Avorn, J.; et al. Strategies to prevent and control the emergence and spread of antimicrobial-resistant microorganisms in hospitals: A challenge to hospital leadership. J. Am. Med Assoc. (JAMA) 1996, 275, 234-240. [CrossRef]

22. Boggan, J.C.; Navar-Boggan, A.M.; Jhaveri, R. Pediatric-specific antimicrobial susceptibility data and empiric antibiotic selection. Pediatrics 2012, 130, e615-e622. [CrossRef]

23. Bielicki, J.A.; Sharl, M.; Johnson, A.P.; Henderson, K.L.; Cromwell, D.A.; Antibiotic Resistance and Prescribing in European Children Project; Berger, C.; Esposito, S.; Danieli, E.; Tenconi, R.; et al. Selecting appropriate empirical antibiotic regimens for paediatric bloodstream infections: Application of a Bayesian decision model to local and pooled antimicrobial resistance surveillance data. J. Antimicrob. Chemother. 2016, 71, 794-802. [CrossRef]

24. Koningstein, M.; van der Bij, A.K.; de Kraker, M.E.; Monen, J.C.; Muilwijk, J.; de Greeff, S.C.; Geerlings, S.E.; Leverstein-van Hall, M.A.; ISIS-AR Study Group. Recommendations for the empirical treatment of complicated urinary tract infections using surveillance data on antimicrobial resistance in the Netherlands. PLoS ONE 2014, 9, e86634. [CrossRef]

25. Mehl, A.; Åsvold, B.O.; Kümmel, A.; Lydersen, S.; Paulsen, J.; Haugan, I.; Solligård, E.; Damås, J.K.; Harthug, S.; Edna, T.H. Trends in antimicrobial resistance and empiric antibiotic therapy of bloodstream infections at a general hospital in Mid-Norway: A prospective observational study. BMC Infect. Dis. 2017, 17, 116.

26. Krumperman, P.H. Multiple antibiotic resistance indexing of Escherichia coli to identify high-risk sources of fecal contamination of foods. Appl. Environ. Microbiol. 1983, 46, 165-170. [CrossRef]

27. Johnson, A.P. Surveillance of antibiotic resistance. Philos. Trans. R. Soc. Biol. Sci. 2015, 370. [CrossRef] 
28. Rossolini, G.; Mantengoli, E. Antimicrobial resistance in Europe and its potential impact on empirical therapy. Clin. Microbiol. Infect. 2008, 14, 2-8. [CrossRef]

29. Agodi, A.; Barchitta, M.; Quattrocchi, A.; Maugeri, A.; Aldisio, E.; Marchese, A.E.; Mattaliano, A.R.; Tsakris, A. Antibiotic trends of Klebsiella pneumoniae and Acinetobacter baumannii resistance indicators in an intensive care unit of Southern Italy, 2008-2013. Antimicrob. Resist. Infect. Control 2015, 4, 43. [CrossRef]

30. Seber, G.A.; Lee, A.J. Linear Regression Analysis; John Wiley \& Sons: Hoboken, NJ, USA, 2012; Volume 329.

31. Brockwell, P.J.; Davis, R.A. Introduction to Time Series and Forecasting; Springer: Berlin/Heidelberg, Germany, 2016.

32. Box, G.E.; Jenkins, G.M.; Reinsel, G.C.; Ljung, G.M. Time Series Analysis: Forecasting and Control; John Wiley \& Sons: Hoboken, NJ, USA, 2015.

33. Public Health England. UK Standards for Microbiology Investigations: Quality and Consistency in Clinical Laboratories; PHE: London, UK, 2014.

34. British Society for Antimicrobial Chemotherapy. BSAC Methods fro Antimicrobial Susceptibility Testing; Version 12; BSAC: London, UK, 2013.

35. Seabold, S.; Perktold, J. Statsmodels: Econometric and statistical modeling with python. In Proceedings of the 9th Python in Science Conference, Austin, TX, USA, 28 June-3 July 2010.

36. McKinney, W. Data Structures for Statistical Computing in Python. In Proceedings of the 9th Python in Science Conference, Austin, TX, USA, 28 June-3 July 2010; pp. 51-56.

37. Waskom, M.; Botvinnik, O.; O’Kane, D.; Hobson, P.; Lukauskas, S.; Gemperline, D.C.; Augspurger, T.; Halchenko, Y.; Cole, J.B.; Warmenhoven, J.; et al. mwaskom/seaborn: v0.8.1 (September 2017). Zenodo 2017. [CrossRef]

38. Hunter, J.D. Matplotlib: A 2D graphics environment. Comput. Sci. Eng. 2007, 9, 90-95. [CrossRef]

39. Farrell, D.; Morrissey, I.; De Rubeis, D.; Robbins, M.; Felmingham, D. A UK multicentre study of the antimicrobial susceptibility of bacterial pathogens causing urinary tract infection. J. Infect. 2003, 46, 94-100. [CrossRef] [PubMed]

40. Kahlmeter, G.; Åhman, J.; Matuschek, E. Antimicrobial resistance of Escherichia coli causing uncomplicated urinary tract infections: A European update for 2014 and comparison with 2000 and 2008. Infect. Dis. Ther. 2015, 4, 417-423. [CrossRef]

41. Public Health England. English Surveillance Programme for Antimicrobial Utilisation and Resistance (ESPAUR); Annual Report 2015; PHE: London, UK, 2015.

42. Bean, D.C.; Krahe, D.; Wareham, D.W. Antimicrobial resistance in community and nosocomial Escherichia coli urinary tract isolates, London 2005-2006. Ann. Clin. Microbiol. Antimicrob. 2008, 7, 13. [CrossRef]

43. Public Health Wales. Antibacterial Resistance in Wales: 2006-2015; PHW: Cardiff, UK, 2015.

44. Public Health England. English Surveillance Programme for Antimicrobial Utilisation and Resistance (ESPAUR); Annual Report 2014; PHE: London, UK, 2014.

45. Department of Health \& Public Health England. Antimicrobial Resistance Empirical and Statistical Evidence-Based; PHE: London, UK, 2016.

46. Fluit, A.C.; Jones, M.E.; Schmitz, F.J.; Acar, J.; Gupta, R.; Verhoef, J. Antimicrobial susceptibility and frequency of occurrence of clinical blood isolates in Europe from the SENTRY antimicrobial surveillance program, 1997 and 1998. Clin. Infect. Dis. 2000, 30, 454-460. [CrossRef]

47. Wisplinghoff, H.; Bischoff, T.; Tallent, S.M.; Seifert, H.; Wenzel, R.P.; Edmond, M.B. Nosocomial bloodstream infections in US hospitals: Analysis of 24,179 cases from a prospective nationwide surveillance study. Clin. Infect. Dis. 2004, 39, 309-317. [CrossRef]

48. Dancer, S.J. Importance of the environment in meticillin-resistant Staphylococcus aureus acquisition: The case for hospital cleaning. Lancet Infect. Dis. 2008, 8, 101-113. [CrossRef]

49. Rammelkamp, C.H.; Maxon, T. Resistance of Staphylococcus aureus to the Action of Penicillin. Exp. Biol. Med. 1942, 51, 386-389. [CrossRef]

50. Johnson, A.P.; Pearson, A.; Duckworth, G. Surveillance and epidemiology of MRSA bacteraemia in the UK. J. Antimicrob. Chemother. 2005, 56, 455-462. [CrossRef] [PubMed]

51. Johnson, A.P.; Davies, J.; Guy, R.; Abernethy, J.; Sheridan, E.; Pearson, A.; Duckworth, G. Mandatory surveillance of methicillinresistant Staphylococcus aureus (MRSA) bacteraemia in England: The first 10 years. J. Antimicrob. Chemother. 2012, 67, 802-809. [CrossRef] [PubMed]

52. Gerver, S.; Mihalkova, M.; Abernethy, J.; Bou-Antoun, S.; Nsonwu, O.; Kauser, S.; Wasti, S.; Apraku, D.; Davies, J.; Hope, R. Annual Epidemiological Commentary: Mandatory MRSA, MSSA and E. coli Bacteraemia and C. Difficile Infection; Public Health England: London, UK, 2014.

53. Hernandez, B.; Herrero, P.; Rawson, T.M.; Moore, L.S.P.; Charani, E.; Holmes, A.H.; Georgiou, P. Data-driven Web-based Intelligent Decision Support System for Infection Management at Point-Of-Care: Case-Based Reasoning Benefits and Limitations. In Proceedings of the 10th International Joint Conference on Biomedical Engineering Systems and Technologies, Porto, Portugal, 21-23 February 2017; Volume 5, pp. 119-127.

54. Hernandez, B.; Herrero, P.; Rawson, T.M.; Moore, L.S.P.; Evans, B.; Toumazou, C.; Holmes, A.H.; Georgiou, P. Supervised learning for infection risk inference using pathology data. BMC Med. Inform. Decis. Mak. 2017, 17, 168. [CrossRef] [PubMed]

55. Hernandez, B. Data-Driven Web-Based Intelligent Decision Support System for Infection Management at Point of Care. Ph.D. Thesis, Imperial College London, London, UK, 2019. [CrossRef] 
56. Rawson, T.M.; Hernandez, B.; Moore, L.S.; Herrero, P.; Charani, E.; Ming, D.; Wilson, R.C.; Blandy, O.; Sriskandan, S.; Gilchrist, M.; et al. A real-world evaluation of a Case-Based Reasoning algorithm to support antimicrobial prescribing decisions in acute care. Clin. Infect. Dis. 2021, 72, 2103-2111. [CrossRef]

57. Rawson, T.M.; Hernandez, B.; Moore, L.S.P.; Blandy, O.; Herrero, P.; Gilchrist, M.; Gordon, A.; Toumazou, C.; Sriskandan, S.; Georgiou, P.; et al. Supervised machine learning for the prediction of infection on admission to hospital: A prospective observational cohort study. J. Antimicrob. Chemother. 2018, 74, 1108-1115. [CrossRef] 